\title{
Biodeterioração e propriedades colorimétricas da madeira de Maclura tinctoria
}

\author{
Danilo Leão de Freitas ${ }^{1}$ Douglas Edson Carvalho ${ }^{2 *}$ Clóvis Roberto Haselein ${ }^{1}$ Cristiani Pedrazzi ${ }^{1}$ \\ Rodrigo Coldebella ${ }^{1}$ Henrique Weber Dalla Costa ${ }^{1}$ Sabrina Finatto Machado ${ }^{1}$
}

${ }^{1}$ Universidade Federal de Santa Maria, Av. Roraima, 1000, Camobi CEP: 97105900, Santa Maria, RS, Brasil

${ }^{2}$ Universidade Federal do Paraná, Av. Pref. Lothário Meissner, 632, Jardim Botânico, Campus III, CEP 80210-170, Curitiba, PR, Brasil

\section{Original Article \\ *Corresponding author: douglasedsoncarvalho@gma il.com \\ Keywords: \\ CIELab \\ Gloeophyllum trabeum \\ Pycnoporus sanguineus \\ Natural resistance \\ Tajuva \\ Palavras-chave: \\ CIELab}

Gloeophyllum trabeum

Pycnoporus sanguineus

Resistência natural

Tajuva

Received in

2020/05/05

Accepted on

2020/07/12

Published in

2020/11/02

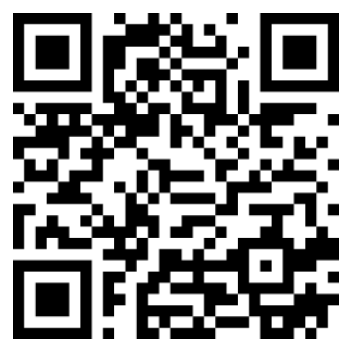

DOI:

http://dx.doi.org/10.34062/af s.v6i2.5621

\section{(cc) BY}

RESUMO: Os fungos apodrecedores são agentes bióticos que, além da deterioração, causam alteração de cor e estão entre os maiores desvalorizadores de produtos à base de madeira. Nesse contexto, o trabalho teve como objetivo avaliar a perda de massa e a alteração da cor do cerne e do alburno da madeira de tajuva (Maclura tinctoria), submetida a ensaio de apodrecimento acelerado com os fungos Gloeophyllum trabeum (podridão parda) e Pycnoporus sanguineus (podridão branca). As amostras foram avaliadas quanto à resistência natural pela análise da perda de massa após exposição aos fungos apodrecedores e pela mudança da cor pelo uso do sistema de medições CIELab. Assim, por meio das variáveis luminosidade $(\mathrm{L})$, coordenadas cromáticas ( $a^{*}$ e $\left.b^{*}\right)$, saturação $(C)$, ângulo de tinta $\left(\mathrm{h}^{*}\right)$ e calculado a variação total da cor $\left(\Delta \mathrm{E}^{*}\right)$. O cerne de Maclura tinctoria apresentou elevada resistência aos fungos apodrecedores com perdas de massa de $1,52 \%$ para Pycnoporus sanguineus e de 0,96\% para Gloeophyllum trabeum. O fungo Pycnoporus sanguineus deteriorou a madeira de alburno e cerne com maior intensidade e alterou a cor natural de brancoacinzentado/ amarelo para amarelo-claro. A variação total na cor da madeira foi considerável e com maior representatividade para o cerne.

\section{Biodeterioration and colorimetric properties of Maclura tinctoria wood}

\begin{abstract}
Rotting fungi are biotic agents that, in addition to deterioration, cause color change and are among the largest devaluators of wood-based products. In this context, the aim of the study was to evaluate the loss of mass and the change in the color of the heartwood and sapwood of tajuva wood (Maclura tinctoria), submitted to an accelerated rotting test with the fungi Gloeophyllum trabeum (brown rot) and Pycnoporus sanguineus (white rot). The samples were evaluated for natural resistance by analysis of mass loss after exposure to rotting fungi and by color change using the CIELab measurement system. Thus, by means of the variables luminosity (L), chromatic coordinates $\left(\mathrm{a}^{*}\right.$ and $\left.\mathrm{b}^{*}\right)$, saturation $(\mathrm{C})$, hue angle $\left(\mathrm{h}^{*}\right)$ and calculated the total color variation $\left(\Delta \mathrm{E}^{*}\right)$. Heartwood of Maclura tinctoria showed high resistance to rotting fungi with mass losses of $1.52 \%$ for Pycnoporus sanguineus and $0.96 \%$ for Gloeophyllum trabeum. The fungus Pycnoporus sanguineus has deteriorated the sapwood and heartwood with greater intensity and changed the natural color from greyish white/ yellow to light yellow. The total variation in the color of the wood was considerable and with greater representativity for the heartwood.
\end{abstract}




\section{Introdução}

A cor é uma característica importante para a identificação, classificação e uso da madeira como matéria-prima de inúmeros produtos. A Maclura tinctoria, conhecida por tajuva, produz madeira com cerne distinto de cor amarela e alburno esbranquiçado, foi amplamente explorada pela qualidade de sua madeira e a relativa abundância de plantas (Coldebella et al., 2018). A madeira possui fino acabamento, adequada para a fabricação de móveis e assoalhos (Gonzaga, 2006), com cor, brilho e desenho fundamentais na atração visual e determinação da escolha pelo consumidor (Silva et al., 2017).

O aspecto visual é o causador da primeira impressão. Desse modo, a cor é uma característica importante para a seleção de uma madeira específica para o consumidor final (Sivrikaya et al., 2019). Destaca-se a importância da caracterização colorimétrica da madeira, principalmente, quando a aparência superficial é determinante para a sua utilização, refletindo diretamente na sua comercialização (Bonfatti Júnior \& Lengowski, 2018).

A cor da madeira pode ser modificada naturalmente pela alteração da composição química da parede celular. Sob certas condições, os produtos à base de madeira são vulneráveis a danos por fungos que reduzem a vida útil e causam perdas econômicas significativas (Bi et al., 2019). O ataque de fungos altera perceptivelmente a cor da madeira, com intensidades variadas em função da estrutura molecular e durabilidade natural (Vidholdová \& Reinprecht, 2019).

Dentre os fungos que atacam a madeira, os apodrecedores, além de causarem alteração da cor e perda de brilho da madeira, são responsáveis por alterações em suas propriedades físico-mecânicas, que resultam em elevada deterioração do material, consequentemente, perda de massa e resistência mecânica (Stangerlin et al., 2013). A deterioração, causada pela perda de massa, resistência mecânica e mudança da cor original da madeira, pode desvalorizá-la quando está em serviço e comprometer sua vida útil. Porém, o grau de deterioração é decorrente da susceptibilidade ao ataque de cada tipo de madeira (Carvalho et al., 2019).

Em uma mesma espécie, o alburno é mais suscetível à deterioração, já o cerne geralmente possui uma significante resistência natural (Castro et al., 2018), com menor perda de material lenhoso quando atacado por fungos. A perda de massa é um método seguro para a avaliação da resistência a agentes que deterioram a madeira (Carvalho et al., 2016). Nesse sentido, a capacidade natural de madeiras em evitar a perda de massa e alterações na sua coloração frente ao ataque de fungos apodrecedores é fundamental para a indicação e aplicação em usos diversos.
O objetivo deste estudo foi avaliar a perda de massa e as alterações na coloração do cerne e do alburno da madeira de tajuva (Maclura tinctoria), submetida a ensaio de apodrecimento acelerado com os fungos apodrecedores Gloeophyllum trabeum (podridão parda) e Pycnoporus sanguineus (podridão branca).

\section{Material e Métodos \\ Preparo das amostras}

Cinco árvores de tajuva (Maclura tinctoria (L.) Don ex Steud.) utilizadas no estudo. Da região do diâmetro a altura do peito (DAP) foram retiradas, do cerne e do alburno, o total de 60 amostras com dimensões de 25 × 25 x $9 \mathrm{~mm}$ (radial $\mathrm{x}$ tangencial $\mathrm{x}$ longitudinal), as quais foram usadas para determinação da densidade aparente a $12 \%$, perda de massa e colorimetria.

\section{Apodrecimento acelerado}

As amostras da madeira de tajuva (cerne e alburno) foram submetidas a ensaios de apodrecimento acelerado com dois fungos Pycnoporus sanguineus (L.: Fr.) Murr., RLG 10851 (podridão branca) e Gloeophyllum trabeum (Pers. ex Fries) Murr., Mad 617 (podridão parda). Estes foram fornecidos pelo Laboratório de Produtos Florestais - LPF do Serviço Florestal Brasileiro SFB, sediado em Brasília, DF.

Os ensaios de apodrecimento acelerado foram realizados em frascos de vidro redondo com tampa rosqueável, com capacidade de $600 \mathrm{~mL}$. Cada frasco continha $100 \mathrm{~g}$ de latossolo vermelho de horizonte $\mathrm{B}$, com $\mathrm{pH} 6,4$ e $39 \mathrm{~mL}$ de água destilada, determinada em função da capacidade de retenção de água do solo, ajustando a umidade para $130 \%$.

Cada frasco recebeu uma placa suporte de alburno de Pinus sp., que serviu de base para o cultivo dos fungos. Os frascos foram autoclavados a $120^{\circ} \mathrm{C}$ e pressão de $1 \mathrm{~atm}$. durante 50 minutos. Os fungos foram repicados em placas de Petri com meio de cultura líquido contendo extrato de malte, ágar e água destilada. As placas repicadas permaneceram em sala climatizada $\left(27 \pm 1^{\circ} \mathrm{C}\right.$ e 70 $\pm 4 \%$ de umidade relativa) por 20 dias, para que o micélio se desenvolvesse completamente na placa de Petri. A inoculação na placa de suporte foi realizada em capela de fluxo laminar e levada a câmara climatizada para viabilizar o crescimento homogêneo do fungo sobre a superfície da placa de suporte.

As amostras de cerne e alburno de Maclura tinctoria foram esterilizadas e introduzidas assepticamente e individualmente nos frascos com os fungos, com 10 repetições cada. Estas permaneceram em contato com a placa de suporte de Pinus sp. com micélio dos fungos (Figura 1) por 16 semanas (112 dias) em sala climatizada, sob as 
condições já descritas, conforme preconizado pela American Society for Testing and Materials ASTM D-2017 (2005).

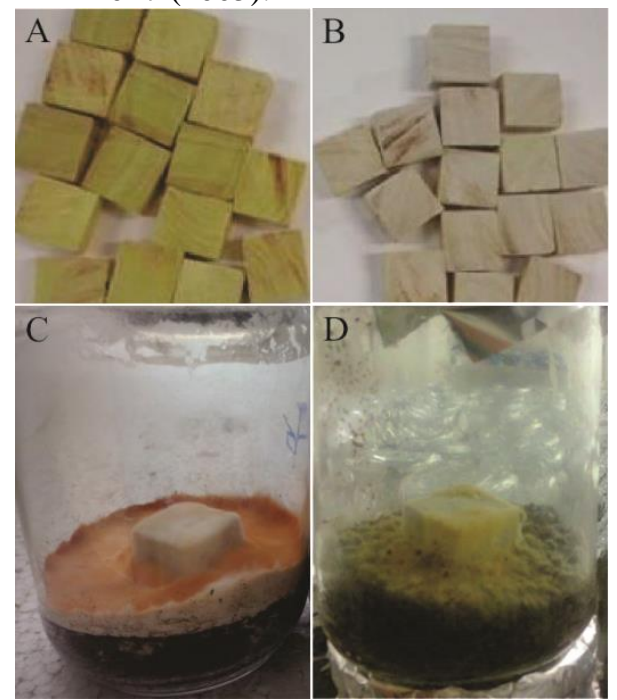

Figura 1. Amostras de cerne (A) e alburno (B) de Maclura trinctoria submetidas a ação do fungo Pycnoporus sanguineus (C) e Gloeophyllum trabeum (D)

Figure 1. Samples of Maclura trinctoria heartwood (A) and sapwood (B) submitted to Pycnoporus sanguineus $(\mathrm{C})$ and Gloeophyllum trabeum fungus.

Decorrido o período de exposição aos fungos, foram removidos os micélios dos fungos acumulados na superfície das amostras e avaliaramse a perda de massa e a alteração colorimétrica. A perda de massa foi calculada pela diferença entre a massa inicial e final da amostra seca em estufa a 63 $\pm 1^{\circ} \mathrm{C}$ até atingir peso constante e classificada conforme indicado pela ASTM D 2017 (2005), (Tabela 1).

Tabela 1. Classes de resistência da madeira a fungos xilófagos, ASTM D 2017 (2005).

\begin{tabular}{ccc}
$\begin{array}{c}\text { Perda de } \\
\text { massa }(\%)\end{array}$ & $\begin{array}{c}\text { Massa } \\
\text { Residual }(\%)\end{array}$ & Classificação \\
\hline $0-10$ & $90-100$ & AR \\
$11-24$ & $76-89$ & R \\
$25-44$ & $56-75$ & MR \\
$>45$ & $<55$ & NR
\end{tabular}

AR: Altamente resistente; R: Resistente; MR: Moderadamente resistente; NR: Não resistente.

\section{Parâmetros colorimétricos}

Os parâmetros colorimétricos foram obtidos com um colorímetro modelo Konica Minolta CR400 , com abertura do sensor de $8 \mathrm{~mm}$. O aparelho foi calibrado para fonte de luz D65 e $10^{\circ}$ de ângulo de observação.

Para a avaliação da variação colorimétrica da madeira, leituras foram realizadas em triplicata na seção transversal de 30 amostras de cada lenho, em condição natural e após o ataque dos fungos. Os parâmetros colorimétricos coletados no sistema CIELab foram a claridade $\mathrm{L}^{*}$; coordenada $\mathrm{a}^{*}$ (vermelho / verde); e coordenada $b^{*}$ (amarelo / azul). Os parâmetros de variação total de cor $(\Delta \mathrm{E})$, a saturação de cor $\left(C^{*}\right)$ e o ângulo de tinta (h) foram calculados conforme a ASTM D2244 (2016).

As cores correspondentes aos grupos de valores dos parâmetros colorimétricos $\left(\mathrm{L}^{*}, \mathrm{a}^{*} \mathrm{e} \mathrm{b}^{*}\right)$ obtidos para cada região da madeira, foram identificadas de acordo com a classificação dos grupos de cores contidos no agrupamento de cluster (Camargos \& Gonçalez, 2001).

\section{Análise de dados}

Os resultados foram analisados quanto à homogeneidade de variância (Bartlett e Hartley's) e normalidade (Kolmogorov - Smirnov). Em seguida foram avaliados pelo emprego da análise de variância (ANOVA) e comparação das médias, quando significativas, pelos testes $t$ (student), para densidade e perda de massa e Tukey, para colorimetria; para ambos $(\mathrm{p}<0,05)$.

\section{Resultados e discussão \\ Biodeterioração da madeira}

Os valores médios de perda de massa foram influenciados pela porção da madeira, cerne ou alburno, e pelo tipo de fungo que a madeira foi exposta. O alburno, quando submetido ao fungo Gloeophyllum trabeum, foi classificado como altamente resistente (Tabela 2). Quando submetido ao ataque do fungo Pycnoporus sanguineus, o alburno foi mais suscetível, enquadrando-se como moderadamente resistente, $\mathrm{O}$ cerne foi qualificado como altamente resistente para ambos os fungos.

A perda de massa para o alburno atacado por Pycnoporus sanguineus (podridão branca) foi cinco vezes maior que a mesma região do lenho atacada por Gloeophyllum trabeum (podridão parda). Em relação à ação do fungo entre cerne e alburno, ambos apresentaram diferença significativa pelo teste T.

Entre o cerne e alburno, a variação da perda de massa ocorreu em função da diferença de substâncias nutritivas e extrativos presentes na madeira de tajuva (Coldebella et al., 2018), alterando assim a resistência natural à organismos xilófagos. A resistência natural de uma madeira está relacionada à presença de extrativos tânicos e outros compostos fenólicos que apresentam toxicidade aos fungos (Carvalho et al., 2015).

A perda de massa para o alburno atacado por Pycnoporus sanguineus (podridão branca) foi cinco vezes maior que a mesma região do lenho atacada por Gloeophyllum trabeum (podridão parda). Em relação à ação do fungo entre cerne e alburno, ambos apresentaram diferença significativa pelo teste $\mathrm{T}$. 
Tabela 2. Valores médios da densidade aparente a $12 \%$ e perda de massa de cerne e alburno de Maclura tinctoria submetida aos fungos Pycnoporus sanguineus e Gloephyllum trabeum.

\begin{tabular}{cccc}
\hline & \multirow{2}{*}{ Densidade aparente $\left(\mathrm{g} \cdot \mathrm{cm}^{-3}\right)$} & \multicolumn{2}{c}{ Perda de massa (\%) } \\
\cline { 3 - 4 } Cerne & $0,67^{\mathrm{ns}}(0,13)$ & $1,52 \mathrm{aA}(1,11)$ & $0,96 \mathrm{aA}(0,46)$ \\
Alburno & $0,70(0,06)$ & $40,44 \mathrm{bA}(11,23)$ & $8,40 \mathrm{bB}(7,23)$ \\
\hline
\end{tabular}

Médias seguidas na vertical por uma mesma letra maiúscula ou na horizontal por uma mesma letra minúscula não diferem (teste de t, $\mathrm{p}>0.05$ ); ns: não significativo (F, p > 0,05). Os valores entre parênteses são os desvios padrões.

Entre o cerne e alburno, a variação da perda de massa ocorreu em função da diferença de substâncias nutritivas e extrativos presentes na madeira de tajuva (Coldebella et al., 2018), alterando assim a resistência natural à organismos xilófagos. A resistência natural de uma madeira está relacionada à presença de extrativos tânicos e outros compostos fenólicos que apresentam toxicidade aos fungos (Carvalho et al., 2015).

Para a perda de massa do alburno, verificouse diferença significativa entre os fungos, enquanto no cerne a perda de massa não diferiu o que evidencia a sua maior resistência a ambos os fungos avaliados. O fungo Pycnoporus sanguineus tem como característica degradar intensamente madeiras de folhosas (Curling et al., 2000) e capacidade em degradar a lignina (Ribes et al., 2018), a qual é aproximadamente igual em alburno e cerne de Maclura tinctoria (Coldebella et al., 2018). O cerne é menos afetado pela ação do fungo, em função da maior presença de extrativos, o qual é três vezes superior ao alburno para a Maclura tinctoria (Coldebella et al., 2018).

O alburno é mais susceptível ao ataque de fungos pela elevada disponibilidade de material nutritivo (Gonçalves et al., 2013). No cerne, além da indisponibilidade de substâncias nutritivas, como amido e açucares, a presença de extrativos aumenta sua resistência a organismos xilófagos.

Sabendo que a massa específica aparente das amostras de alburno e de cerne não apresentou diferença significativa (Tabela 2), pode-se afirmar que esta propriedade não interfere na resistência à ação dos fungos, corroborando com demais estudos de resistência de espécies florestais a fungos apodrecedores (Modes et al., 2012; Carvalho et al., 2015).

Portanto, a madeira de cerne de Maclura tinctoria, por apresentar alta resistência biológica é uma alternativa de uso da madeira em ambientes extremos, com propensão a ação de fungos apodrecedores. Uso da madeira de cerne em contato ou próximo ao solo deve ser investigado para elucidar seu desempenho em tais situações.

\section{Parâmetros colorimétricos}

A madeira de alburno atacada pelo fungo de podridão parda (Gloeophyllum trabeum) teve coloração escura (marrom), enquanto a atacada por podridão branca (Pycnoporus sanguineus), coloração clara (esbranquiçada), comportamento semelhante ao descrito por Vivian et al. (2015).

A seletividade do ataque de cada fungo explica as colorações. Fungos de podridão parda degradam principalmente os polissacarídeos, deixando a lignina parcialmente intacta, adquirindo coloração marrom devido ao ataque enzimático (Aguiar \& Ferraz, 2011) e a oxidação da lignina (Monrroy et al., 2011). Fungos causadores de podridão branca degradam todos os componentes lignocelulósicos (Aguiar \& Ferraz, 2011), incluindo a lignina (Ribes et al., 2018), atribuindo aspecto mais claro a madeira.

Os parâmetros colorimétricos foram alterados pelo ataque dos fungos de podridão branca e parda (Tabela 3). Para ambas as regiões da madeira verificaram-se diferenças significativas, quando comparados às amostras testemunhas, com exceções da pigmentação amarela $\left(b^{*}\right)$ e da cromaticidade $\left(\mathrm{C}^{*}\right)$ para a região do alburno, após a exposição ao fungo Gloeophyllum trabeum.

Observou-se redução significativa da luminosidade $\left(\mathrm{L}^{*}\right)$, para as amostras de cerne e alburno expostas aos dois fungos apodrecedores, com predisposição ao escurecimento superficial. As amostras do cerne e do alburno, expostos ao fungo Gloeophyllum trabeum, apresentaram a maior redução de luminosidade, na ordem de $22,94 \%$ e $25,76 \%$, respectivamente.

A alteração dos parâmetros colorimétricos modificou a coloração natural da madeira de amarelo ouro para amarelo-clara e amareloalaranjada, correspondente à ação do fungo Pycnoporus sanguineus e Gloeophyllum trabeum, respectivamente. Em relação ao cerne, quando atacado pelo fungo de podridão branca, observou-se que a superfície da madeira se tornou mais esbranquiçada, apresentando uma redução de sua luminosidade de $\approx 20 \%$.

$\mathrm{Na}$ amostra de cerne atacada pelo Pycnoporus sanguineus a cor amarela foi alterada para uma tonalidade mais clara (amarelo-clara). O fungo Gloeophyllum trabeum, após o deixou a superfície da madeira de cerne com uma coloração mais escura (amarelo-alaranjado). O alburno teve uma coloração inicial branco-acinzentado e posteriormente ao ataque, a madeira ficou com tonalidades amarelo-claro e marrom-avermelhado, respectivamente, para o Pycnoporus sanguineus e Gloeophyllum trabeum. 
Tabela 3. Parâmetros colorimétricos do cerne e alburno da madeira de Maclura tinctoria em condição natural (testemunha) e após o ataque dos fungos Pycnoporus sanguineus (podridão branca) e Gloeophyllum trabeum (podridão parda).

\begin{tabular}{|c|c|c|c|c|c|c|c|}
\hline Madeira & Fungo & $\mathrm{L}^{*}$ & $a^{*}$ & $\mathrm{~b}^{*}$ & $\mathrm{C}^{*}$ & $\mathrm{~h}^{*}$ & Coloração** \\
\hline \multirow{3}{*}{ 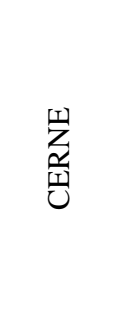 } & Testemunha & $\begin{array}{c}72,67 \mathrm{~A} \\
(2,52)\end{array}$ & $\begin{array}{c}2,98 \mathrm{~A} \\
(1,29)\end{array}$ & $\begin{array}{c}37,51 \mathrm{~A} \\
(2,9)\end{array}$ & $\begin{array}{c}37,65 \mathrm{~A} \\
(2,9)\end{array}$ & $\begin{array}{c}85,73 \mathrm{~A} \\
(2,0)\end{array}$ & Amarelo \\
\hline & $\begin{array}{l}\text { Pycnoporus } \\
\text { sanguineus }\end{array}$ & $\begin{array}{c}58,23 \mathrm{~B} \\
(4,76)\end{array}$ & $\begin{array}{c}10,05 \mathrm{~B} \\
(2,26)\end{array}$ & $\begin{array}{l}42,4 \mathrm{C} \\
(3,49)\end{array}$ & $\begin{array}{c}43,66 \mathrm{~B} \\
(3,19)\end{array}$ & $\begin{array}{l}76,5 \mathrm{~B} \\
(3,49)\end{array}$ & Amarelo-claro \\
\hline & $\begin{array}{c}\text { Gloeophyllum } \\
\text { trabeum }\end{array}$ & $\begin{array}{c}56,00 \mathrm{~B} \\
(6,06)\end{array}$ & $\begin{array}{l}9,23 \mathrm{~B} \\
(2,08)\end{array}$ & $\begin{array}{c}38,94 \mathrm{BC} \\
(7,08)\end{array}$ & $\begin{array}{c}40,11 \mathrm{BC} \\
(7,09)\end{array}$ & $\begin{array}{c}76,41 \mathrm{~B} \\
(3,09)\end{array}$ & $\begin{array}{l}\text { Amarelo- } \\
\text { alaranjado }\end{array}$ \\
\hline \multirow{3}{*}{ 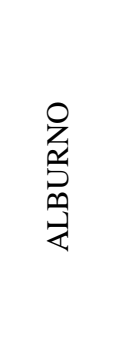 } & Testemunha & $\begin{array}{c}72,85 \mathrm{~A} \\
(1,66)\end{array}$ & $\begin{array}{c}3,20 \mathrm{~A} \\
(0,49)\end{array}$ & $\begin{array}{l}18,5 \mathrm{~A} \\
(2,91)\end{array}$ & $\begin{array}{c}18,84 \mathrm{~A} \\
(2,81)\end{array}$ & $\begin{array}{c}79,31 \mathrm{~A} \\
(2,91)\end{array}$ & $\begin{array}{c}\text { Branco- } \\
\text { acinzentado }\end{array}$ \\
\hline & $\begin{array}{l}\text { Pycnoporus } \\
\text { sanguineus }\end{array}$ & $\begin{array}{c}66,33 \text { B } \\
(2,44)\end{array}$ & $\begin{array}{c}9,10 \mathrm{~B} \\
(1,41)\end{array}$ & $\begin{array}{c}33,96 \mathrm{~B} \\
(2,85)\end{array}$ & $\begin{array}{c}35,18 \mathrm{~B} \\
(3,03)\end{array}$ & $\begin{array}{c}75,04 \mathrm{~B} \\
(1,6)\end{array}$ & Amarelo-claro \\
\hline & $\begin{array}{l}\text { Gloeophyllum } \\
\text { trabeum }\end{array}$ & $\begin{array}{c}54,08 \mathrm{C} \\
(8,47)\end{array}$ & $\begin{array}{c}4,76 \mathrm{C} \\
(0,98)\end{array}$ & $\begin{array}{c}21,04 \mathrm{~A} \\
(3,75)\end{array}$ & $\begin{array}{c}21,59 \mathrm{~A} \\
(3,82)\end{array}$ & $\begin{array}{c}77,29 \mathrm{BC} \\
(1,82)\end{array}$ & $\begin{array}{c}\text { Marrom- } \\
\text { avermelhado }\end{array}$ \\
\hline
\end{tabular}

L*: luminosidade; $a^{*}$ : coordenada cromática vermelho-verde; $b^{*}$ : coordenada cromática amarelo-azul; C: saturação; $h^{*}$ : ângulo de tinta; $\Delta \mathrm{E}^{*}$ : variação total da cor. Para cada madeira, os valores seguidos por letras iguais em cada coluna não diferem (Tukey, $\mathrm{p}>0,05$ ). Os valores entre parênteses são os desvios padrões. **Classificação de cor proposta por Camargos e Gonçalez (2001).

Após ser submetido ao contato com o fungo Gloeophyllum trabeum, a superfície do alburno tornou-se marrom, um dos efeitos da degradação da celulose e hemiceluloses, evidenciando um aspecto de madeira queimada, conferindo o nome de podridão parda. Do mesmo modo, a ação do fungo Pycnoporus sanguineus alterou a coloração da madeira para tons mais claros, coincidindo com o efeito natural da ação do fungo de podridão branca na madeira.

Os fungos de podridão parda decompõem os polissacarídeos, celulose e hemiceluloses da parede celular, enquanto a lignina, é apenas danificada parcialmente (Irbe et al., 2011), resultando em escurecimento gradual e coloração da madeira com uma tonalidade parda. Por outro lado, os fungos causadores da podridão branca atacam, indistintamente, os polissacarídeos quanto à lignina, de modo que a madeira adquire um aspecto mais claro em relação à cor natural (Vivian et al., 2015; Vidholdová \& Reinprecht, 2019).

$\mathrm{O}$ diagrama de $\mathrm{L}^{*} \mathrm{x} \mathrm{C}^{*}$ elucida as alterações ocorridas na madeira de cerne e alburno após a ação dos fungos (Figura 2).

Os valores médios da variação dos parâmetros colorimétricos ( $\mathrm{L}^{*}, \mathrm{a}^{*}$ e $\left.\mathrm{b}^{*}\right)$ e da variação total da cor $(\Delta \mathrm{E})$ do cerne e alburno da madeira de Maclura tinctoria submetida ao ataque pelos fungos apodrecedores encontram-se na Tabela 4.
As maiores variações nas cores foram observadas nas amostras de cerne, para ambos os fungos. No entanto, as variações ocorridas no alburno foram muito semelhantes. Os valores negativos de $\Delta \mathrm{L}^{*}$ e positivos de $\Delta \mathrm{a}^{*}$ e $\Delta \mathrm{b}^{*}$ indicam a redução na luminosidade e o aumento das pigmentações vermelha e amarela, respectivamente.

As maiores reduções em luminosidade foram observadas para as amostras submetidas à ação do fungo Gloeophyllum trabeum, tanto para o cerne, quanto para o alburno. Esse escurecimento tornou-se uma característica marcante nas peças atacadas por esse tipo de fungo, o que é atribuída à remoção dos polissacarídeos da parede celular, celulose e hemiceluloses, por ação enzimática do fungo (Arantes \& Goodell, 2014; Kojima et al., 2016).

Os valores da variação total na coloração $\left(\Delta \mathrm{E}^{*}\right)$ estão de acordo com os encontrados em trabalhos que avaliaram as mudanças de cor de madeiras tropicais sob deterioração biológica, como os realizados por Costa et al. (2011) e Vidholdová \& Reinprecht (2019). Ademais, são ligeiramente inferiores aos observados em avaliações com outras madeiras tropicais (Stangerlin et al., 2013), porém, a variação total é considera muito apreciável, conforme classificação de Hikita et al. (2001). 


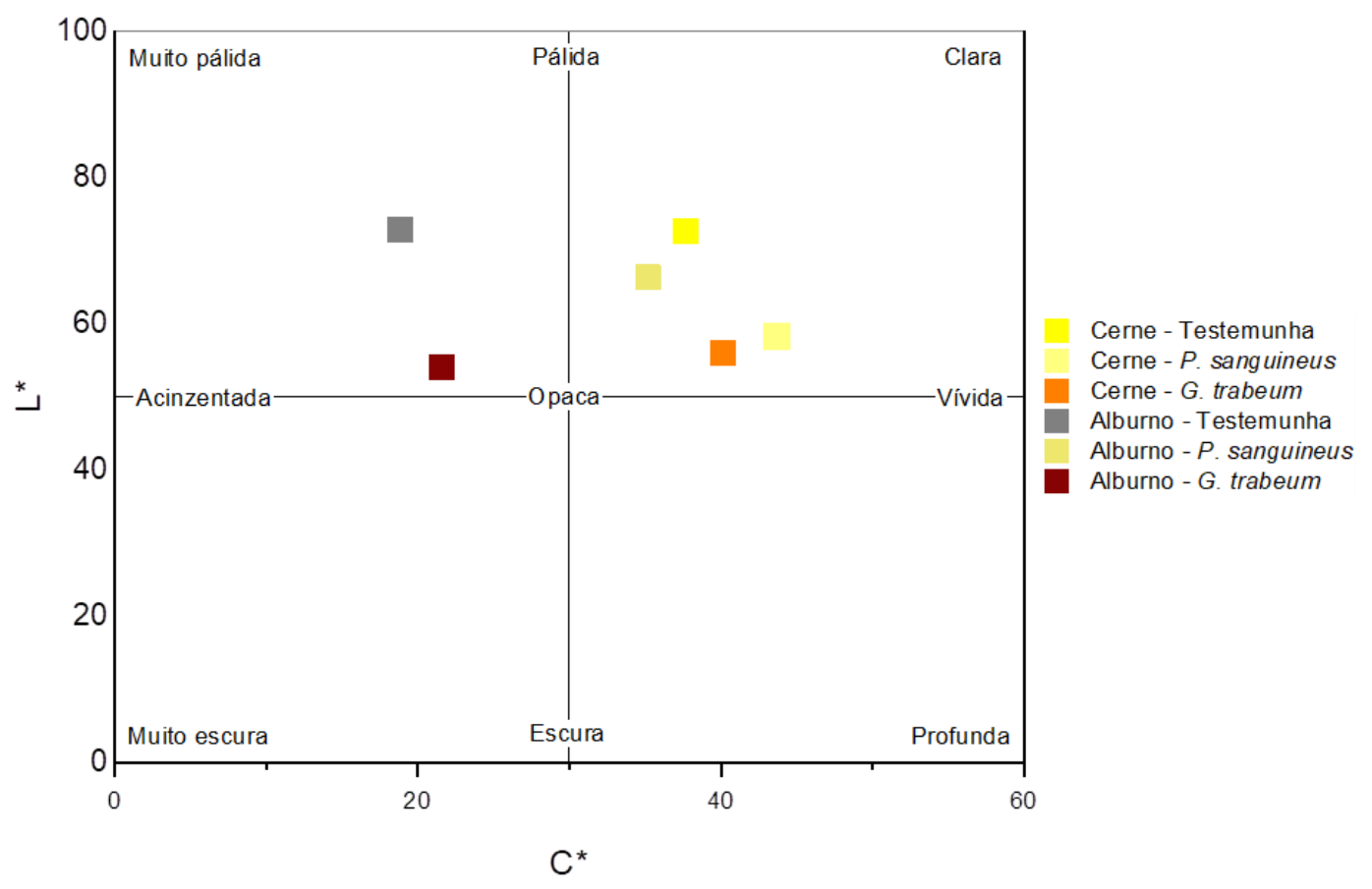

Figura 2. Diagrama de luminosidade (L*) x saturação (C) das amostras de Maclura tinctoria em condição natural e após ação de fungos no cerne e alburno.

Tabela 4. Valores médios da variação dos parâmetros colorimétricos do cerne e alburno da madeira de Maclura tinctoria, submetidos ao ataque dos fungos Pycnoporus sanguineus e Gloeophyllum trabeum

\begin{tabular}{|c|c|c|c|c|c|}
\hline Madeira & Fungo & $\Delta \mathrm{L}^{*}$ & $\Delta \mathrm{a}^{*}$ & $\Delta \mathrm{b}^{*}$ & $\Delta \mathrm{E}^{*}$ \\
\hline \multirow{2}{*}{ 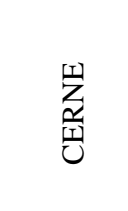 } & $\begin{array}{l}\text { Pycnoporus } \\
\text { sanguineus }\end{array}$ & $\begin{array}{c}-15,57 \mathrm{~A} \\
(2,85)\end{array}$ & $\begin{array}{c}7,44 \mathrm{~A} \\
(2,11)\end{array}$ & $\begin{array}{l}7,44 \mathrm{~A} \\
(2,11)\end{array}$ & $\begin{array}{c}18,49 \mathrm{~A} \\
(2,35)\end{array}$ \\
\hline & $\begin{array}{l}\text { Gloeophyllum } \\
\text { trabeum }\end{array}$ & $\begin{array}{c}-18,94 \mathrm{~A} \\
(5,20)\end{array}$ & $\begin{array}{c}5,85 \mathrm{~A} \\
(2,78)\end{array}$ & $\begin{array}{c}5,24 \mathrm{~A} \\
(2,67)\end{array}$ & $\begin{array}{c}19,10 \mathrm{~A} \\
(2,48)\end{array}$ \\
\hline \multirow{2}{*}{ 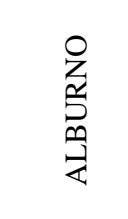 } & $\begin{array}{l}\text { Pycnoporus } \\
\text { sanguineus }\end{array}$ & $\begin{array}{c}-7,80 \mathrm{~B} \\
(3,05)\end{array}$ & $\begin{array}{c}6,45 \mathrm{~A} \\
(1,71)\end{array}$ & $\begin{array}{c}16,02 \mathrm{~B} \\
(3,12)\end{array}$ & $\begin{array}{c}18,19 \mathrm{~A} \\
(2,23)\end{array}$ \\
\hline & $\begin{array}{l}\text { Gloeophyllum } \\
\text { trabeum }\end{array}$ & $\begin{array}{c}-19,56 \mathrm{~A} \\
(9,98)\end{array}$ & $\begin{array}{l}2,07 \mathrm{~B} \\
(1,20)\end{array}$ & $\begin{array}{c}5,19 \mathrm{~A} \\
(4,54)\end{array}$ & $\begin{array}{c}15,32 \mathrm{~B} \\
(0,74)\end{array}$ \\
\hline
\end{tabular}

$\Delta \mathrm{L}^{*}$ : variação da luminosidade; $\Delta \mathrm{a}^{*}$ : variação na coordenada cromática verde-vermelha; $\Delta \mathrm{b}^{*}$ : variação na coordenada cromática amarela-azul; $\Delta \mathrm{E}^{*}$ : variação total da coloração. Os valores seguidos por letras iguais em cada coluna não diferem (Tukey, $\mathrm{p}>0,05$ ). Os valores entre parênteses são os desvios padrões.

No contexto geral, alterações de cor são indesejadas, pois estão associadas a padrões estéticos, quanto ao uso da madeira. Por outro lado, a disposição da madeira a ação intencional de fungos pode resultar em baixa perda de massa e alterar a coloração, como no cerne de Maclura tinctoria, o que pode ser interessante esteticamente para usos em artefatos decorativos. Mas, nessa situação, a ação dos fungos deve ser realizada antes do uso da madeira e interrompida de forma a cessar o desenvolvimento do fungo.

\section{Conclusões}

A madeira de Maclura tinctoria apresentou elevada resistência natural, com destaque para a região do cerne.

A densidade não influenciou na resistência natural da madeira de Maclura tinctoria.

$\mathrm{O}$ alburno e cerne foram deteriorados com maior intensidade pelo fungo Pycnoporus sanguineus, que alterou a cor natural para amareloclaro. 
O fungo Gloeophyllum trabeum atribuiu uma coloração semelhante a tons pardos ao alburno e cerne.

A variação total na cor da madeira foi considerável e mais representativa para o cerne.

\section{Referências}

Aguiar A, Ferraz A (2011) Mecanismos envolvidos na biodegradação de materiais lignocelulósicos e aplicações tecnológicas correlatas. Química Nova, 34(10): $1729-1738$. doi:10.1590/S010040422011001000006 .

American Society For Testing And Materials ASTM D2017: Standard test method for accelerated laboratory test of natural decay resistance of wood. Annual Book of ASTM Standards, West Conshohocken , 2005.

American Society For Testing And Materials ASTM D2244: Standard Practice for Calculation of Color Tolerances and Color Differences from Instrumentally Measured Color Coordinates, Annual Book of ASTM Standards, West Conshohocken, 2016.

Arantes V, Goodell B (2014) Current understanding of brown-rot fungal biodegradation mechanisms: a review. In Schultz, T.P.; Goodell, B.; Nicholas, D.D. Deterioration and protection of sustainable biomaterials. American Chemical Society, Washington, 1158: 3-21. doi:10.1021/bk-20141158.ch001

Bi Z, Yang F, Lei, Y, Morrell JJ, Yan L (2019) Identification of antifungal compounds in konjac flying powder and assessment against wood decay fungi. Industrial Crops and Products, 140:1-7. doi:10.1016/j.indcrop.2019.111650

Bonfatti Júnior EA, Lengowski EC (2018) Colorimetria aplicada à ciência e tecnologia da madeira. Pesquisa Florestal Brasileira, 38: 1-13. doi:10.4336/2018.pfb.38e201601394

Camargos JAA, Gonçalez JC (2001) Colorimetria aplicada como instrumento na elaboração de uma tabela de cores de madeira. Brasil Florestal, 71: 3041. https://core.ac.uk/download/pdf/33542076.pdf. 04 Fev. 2020

Carvalho DE, Santini EJ, Gouveia FN, Rocha MP (2015) Resistência natural de quatro espécies florestais submetidas a ensaio com fungos apodrecedores. Floresta e Ambiente, 22(2): 271276. doi:10.1590/2179-8087.105914

Carvalho DE, Santini EJ, Martins APM, Susin F, Freitas LS, Talgatti M (2016) Natural durability of Eucalyptus dunnii Maiden, Eucalyptus robusta Sm.,
Eucalyptus tereticornis Sm. and Hovenia dulcis Thunb. wood in field and forest environment. Revista Árvore, 40(2): 363-370. doi:10.1590/010067622016000200019

Carvalho DE, Rocha MP, Klitzke RJ, De Cademartori PHG, Juízo CGF, Martins APM (2019) Resistência e rigidez da madeira de espécies florestais submetidas a campo de apodrecimento. Advances in Forestry Science, 6(1): 535-540. doi:10.34062/afs.v6i1.7242

Castro VG, Guimarães PP, Carvalho DE, Souza GO, Brochini GG, Azambuja RR, Rosa TS, Rocha MP, Loiola PL, Dias PC, Pereira KTO (2018) Deterioração e preservação da madeira. Mossoró: EdUFERSA.

Coldebella R, Giesbrecht BM. Saccol AFO. Gentil M, Pedrazzi C (2018) Propriedades físicas e químicas da madeira de Maclura tinctoria (L.) D.Don ex Steud. Ciência da Madeira, 9(1): 54-61. doi:10.15210/cmad.v9i1.10818

Costa MA, Costa AF, Pastore TCM, Braga JWB, Gonçalez JC (2011) Caracterização do ataque de fungos apodrecedores de madeiras através da colorimetria e da espectroscopia de infravermelho. Ciência Florestal, 21(3): 67-577. doi:10.5902/198050983814

Curling S, Winandy JE, Clausen CA (2000) An experimental method to simulate incipiente decay of wood by basidiomycete fungi. In: The International Research Group on Wood Preservation; Section 2, Test methodology and assessment; IRG/WP 00-20200, 31, 2000, Kona.13p.

https://www.fs.usda.gov/treesearch/pubs/5698. 10 Set. 2019.

Gonçalves FG, Pinheiro DTC, Paes JB. Carvalho AG, Oliveira GL (2013) Durabilidade natural de espécies florestais madeireiras ao ataque de cupim de madeira seca. Floresta e Ambiente, 20(1): 110116. doi:10.4322/floram.2012.063

Gonzaga AL (2006) Madeira: uso e conservação. Brasília, Iphan/Monumenta, 246p. (Cadernos Técnicos, 6). http://portal.iphan.gov.br/uploads/ publicacao/CadTec6_MadeiraUsoEConservacao.pd f. 23 Out. 2019.

Hikita Y, Toyoda T, Azuma M (2001) Weathering testing of timber: discoloration. In: Imamura Y. High performance utilization of wood for outdoor uses. Kyoto: Press-Net. 
Irbe I, Andersone I. Andersons B. Noldt G. Dizhbite T, Kurnosova N, Nuopponen M, Stewart D (2011) Characterisation of the initial degradation stage of Scots pine (Pinus sylvestris L.) sapwood after attack by brow-rot fungus Coniophora puteana. Biodegradation, 22(4): 719-728. doi:10.1007/s10532-010-9449-6

Kojima Y, Várnai A, Ishida T, Sunagawa N, Petrovic DM, Igarashi K, Jellison J, Goodell B, Alfredsen G, Westereng B, Eijsink VGH, Yoshida M (2016) Characterization of an LPMO from the brown-rot fungus Gloephyllum trabeum with broad xyloglucan specificity, and its action on cellulosexyloglucan complexes. Applied Environmental Microbiology, 82(22): 6557-6572. doi:10.1128/AEM.01768-16

Modes KS, Lazarotto M, Beltrame R, Vivian MA, Santini EJ, Muniz FB (2012) Resistência natural das madeiras de sete espécies florestais ao fungo Pycnoporus sanguineus causador da podridãobranca. Cerne, 18(3): 407-411. doi:10.1590/S010477602012000300007

Monrroy M, Ortega I, Ramírez M, Baeza J, Freer J (2011) Structural Change in Wood by Brown Rot Fungi and Effect on Enzymatic Hydrolysis. Enzyme Microb Technol. 49(5):472-477. doi: 10.1016/j.enzmictec.2011.08.004.

Ribes DD, Zanatta P, Gallio E, Lourençon T, Beltrame R, Pedrazzi C, Gatto DA (2018) Utilização do fungo Pycnoporus sanguineus para biopolpação de madeira. Revista Matéria, 23(4). doi:10.1590/s1517-707620180004.0568

Silva RAF, Setter C, Mazette SS, Melo RR, Stangerlin DM (2017) Colorimetria da madeira de trinta espécies tropicais. Ciência da Madeira, 8(1): 36-41. doi:10.15210/cmad.v8i1.9686

Sivrikayaa H, Tesařováb D, Jeřábkováb E, Can A (2019) Color change and emission of volatile organic compounds from Scots pine exposed to heat and vacuum-heat treatment. Journal of Building Engineering, 26:1-7. doi:10.1016/j.jobe.2019.100918

Stangerlin DM, Costa AF, Gonçalez JC, Pastore TCM, Garlet A (2013) Monitoramento da biodeterioração da madeira de três espécies amazônicas pela técnica da colorimetria. Acta Amazônica, 43(4): 429-438. doi:10.1590/S004459672013000400004

Vidholdová Z, Reinprecht L (2019) The colour of tropical woods influenced by brown rot. Forests, 10(4): 322. doi:10.3390/f10040322
Vivian MA, Santini EJ, Modes KS, Garlet A. Morais WWC (2015) Resistência biológica da madeira tratada de Eucalyptus grandis e Eucalyptus cloeziana a fungos apodrecedores em ensaios de laboratório. Ciência Florestal, 25(1): 175-183. doi:/10.5902/1980509817475. 